PROCEEDINGS OF THE AMERICAN MATHEMATICAL SOCIETY

Volume 125, Number 5, May 1997, Pages 1283-1291

S 0002-9939(97)03697-6

\title{
QUASITILTED ALGEBRAS ADMIT A PREPROJECTIVE COMPONENT
}

\author{
FLAVIO U. COELHO AND DIETER HAPPEL
}

(Communicated by Ken Goodearl)

\begin{abstract}
Quasitilted algebras are generalizations of tilted algebras. As a main result we show here that the Auslander-Reiten quiver of such an algebra has a preprojective component
\end{abstract}

Let $k$ be an algebraically closed field and $\Lambda$ be a finite-dimensional $k$-algebra. We denote by mod $\Lambda$ the category of finitely generated left $\Lambda$-modules. By $\Gamma_{\Lambda}$ we denote the Auslander-Reiten quiver of $\Lambda$. Recall that the vertices of $\Gamma_{\Lambda}$ correspond to the isomorphism classes of indecomposable finitely generated $\Lambda$-modules. The number of arrows from an indecomposable $\Lambda$-module $X$ to an indecomposable $\Lambda$ module $Y$ is the dimension of the $k$-vector space $\operatorname{rad}(X, Y) / \operatorname{rad}^{2}(X, Y)$, where $\operatorname{rad}(-,-)$ denotes the Jacobson radical of $\bmod \Lambda$. We denote by $\tau X=D \operatorname{Tr} X$ the Auslander-Reiten translate of the indecomposable $\Lambda$-module $X$. This is defined for each indecomposable module, and in case $X$ is non-projective the translate $\tau X$ will be indecomposable and non-injective. Dually there is defined $\tau^{-} X=\operatorname{Tr} D X$. A connected component $\mathcal{P}$ of $\Gamma_{\Lambda}$ is called a preprojective component if $\mathcal{P}$ does not contain an oriented cycle and each $X \in \mathcal{P}$ is of the form $\tau^{-r} P$ for some $r \in \mathbb{N}$ and an indecomposable projective $\Lambda$-module $P$. For details see [ARS]. The existence of preprojective components has been established for various classes of algebras such as tilted algebras [St] or algebras satisfying the separation condition [B]. It is well known that tilted algebras may have several preprojective components. One of the important features of an indecomposable module $X$ lying in a preprojective component is that $X$ is homologically trivial, i.e. $\operatorname{Ext}_{\Lambda}^{i}(X, X)=0$ for $i>0$ and $\operatorname{End}_{\Lambda} X=k$ and that its isomorphism class is uniquely determined by the composition factors. Also these modules can be constructed easily by the socalled knitting procedure.

Quasitilted algebras have been introduced and investigated in [HRS2]. Recall that a finite-dimensional $k$-algebra $\Lambda$ is called a quasitilted algebra if there exist a hereditary abelian $k$-category $\mathcal{H}$ and a tilting object $T \in \mathcal{H}$ such that $\Lambda=\operatorname{End}_{\mathcal{H}} T$. In this article we will not work with this definition but rather with the homological characterization established in [HRS2]. We will use the following notation. For

Received by the editors March 31, 1995 and, in revised form, November 10, 1995.

1991 Mathematics Subject Classification. Primary 16G10, 16E10.

Key words and phrases. Preprojective components, quasitilted algebras.

This work was done when the first named author was visiting the Chemnitz University under the financial support of FAPESP and CNPq, Brazil. He would like to thank the second author for the kind hospitalitly during his stay. The authors also would like to thank J. A. de la Peña for pointing out some inaccuracies in an earlier version. 
$X \in \bmod \Lambda$ we denote by $\operatorname{pd}_{\Lambda} X\left(\operatorname{resp} . \operatorname{id}_{\Lambda} X\right)$ the projective dimension (resp. the injective dimension) of $X$, and we denote by gl.dim $\Lambda$ the global dimension of $\Lambda$. The algebra $\Lambda$ is quasitilted if and only if gl.dim $\Lambda=2$, and for each indecomposable $\Lambda$-module $X$ we have either $\operatorname{pd}_{\Lambda} X \leq 1$ or $\operatorname{id}_{\Lambda} X \leq 1$.

As a main result we will show that the Auslander-Reiten quiver of a quasitilted algebra always has a preprojective component. Since tilted algebras are known to have a preprojective component we can restrict to the case of quasitilted algebras which are not tilted. In this situation we can use results from [CS], where also other aspects of the structure of the Auslander-Reiten quiver of a quasitilted algebra are considered. We also refer to [S] for recent results on tame quasitilted algebras.

The key idea of the proof is to investigate in detail conditions on a module $M$ over a quasitilted algebra $\Lambda$ such that the one-point extension algebra $\Lambda[M]$ is again quasitilted. This will extend results obtained in [HRS2]. The results obtained here will then allow us to make use of a result in [DP].

In the first section we start by recalling some preliminary facts. The second section contains the proof of the theorem, while in section 3 we will present some examples. We denote the composition of morphisms $f: X \rightarrow Y$ and $g: Y \rightarrow Z$ in a given category $\mathcal{K}$ by $f g$. The following notation will be useful. Let $M, N, X$ be $\Lambda$-modules and $f: M \rightarrow X$ a map. Then we will denote by $\hat{f}: M \oplus N \rightarrow X$ the map whose restriction to $M$ is $f$ and whose restriction to $N$ is zero. For unexplained terminology and for some representation-theoretic background we refer to [ARS] and $[R]$.

\section{Preliminaries}

In this section we will recall some basic facts on quasitilted algebras from [HRS2]. Moreover, we will study one-point extensions of quasitilted algebras.

Let $\Lambda$ be a finite-dimensional $k$-algebra. A path in $\bmod \Lambda$ is a sequence $\left(X_{0}, \ldots, X_{t}\right)$ of indecomposable $\Lambda$-modules $X_{i}$ for $0 \leq i \leq t$, such that there is a map $0 \neq f_{i} \in \operatorname{rad}\left(X_{i}, X_{i+1}\right)$ for $0 \leq i<t$. In this case we write $X_{0} \preccurlyeq X_{t}$ and say that $X_{0}$ is a predecessor of $X_{t}$ and that $X_{t}$ is a successor of $X_{0}$. If $t \geq 1$ and $X_{0} \simeq X_{t}$ we say that the path is a cycle. If $t=1$ and $X_{0} \simeq X_{1}$ we say that the path is a short cycle. We say that the path $\left(X_{0}, \ldots, X_{t}\right)$ is sectional if $X_{i-1} \neq \tau X_{i+1}$ for $0<i<t$. If $\left(X_{0}, \ldots, X_{t}\right)$ is a path, we say that a path $\left(Y_{0}, \ldots, Y_{s}\right)$ is a refinement of $\left(X_{0}, \ldots, X_{t}\right)$ if there is an order-preserving function $\pi:\{0, \ldots, t\} \rightarrow\{0, \ldots, s\}$ such that $X_{i}=Y_{\pi(i)}, \pi(0)=0, \pi(t)=s$. A refinement $\left(Y_{0}, \ldots, Y_{s}\right)$ of a path $\left(X_{0}, \ldots, X_{t}\right)$ is said to be a refinement of irreducible maps if there is an irreducible map from $Y_{i}$ to $Y_{i+1}$ for all $0 \leq i<s$, or equivalently $\operatorname{rad}\left(Y_{i}, Y_{i+1}\right) / \operatorname{rad}^{2}\left(Y_{i}, Y_{i+1}\right) \neq 0$ for all $0 \leq i<s$.

Following $[\mathrm{HR}]$ we say that a module $M$ is directing provided there do not exist indecomposable summands $M_{1}$ and $M_{2}$ of $M$, and an indecomposable non-projective module $W$ such that $M_{1} \preccurlyeq \tau W$ and $W \preccurlyeq M_{2}$. We refer to [HR] for a further study of directing modules.

The following subcategories of $\bmod \Lambda$ are useful. We denote by ind $\Lambda$ the full subcategory of $\bmod \Lambda$ containing a chosen set of representatives of the isomorphism classes of indecomposable $\Lambda$-modules. We denote by $\mathcal{L}=\mathcal{L}(\Lambda)$ the full subcategory of ind $\Lambda$ containing those indecomposable modules $X$ such that every predecessor $Y$ of $X$ satisfies $\operatorname{pd}_{\Lambda} Y \leq 1$. Dually, we denote by $\mathcal{R}=\mathcal{R}(\Lambda)$ the full subcategory of ind $\Lambda$ containing those indecomposable modules $X$ such that every successor $Y$ of $X$ 
satisfies $\operatorname{id}_{\Lambda} Y \leq 1$. Using this we have the following characterization of quasitilted algebras [HRS2], Theorem 1.14.

Theorem 1.1. The following are equivalent for a finite-dimensional $k$-algebra $\Lambda$.

(i) $\Lambda$ is quasitilted.

(ii) $\mathcal{L}$ contains all indecomposable projective modules.

(iii) $\mathcal{R}$ contains all indecomposable injective modules.

(iv) Any path in mod $\Lambda$ starting in an indecomposable injective module and ending in an indecomposable projective module has a refinement of irreducible maps, and any such refinement is sectional.

We will also need the following lemma. A proof is basically contained in [HRS1] or [HRS2].

Lemma 1.2. Let $\Lambda$ be a quasitilted algebra and $\left(X_{0}, \ldots, X_{t}\right)$ be a path contained in ind $\Lambda$. If $X_{0}$ belongs to $\mathcal{R}$ or if $X_{t}$ belongs to $\mathcal{L}$, then there exist an indecomposable module $Y$ and nonzero maps $X_{0} \rightarrow Y$ and $Y \rightarrow X_{t}$. In particular, an indecomposable $\Lambda$-module $X$ belongs to a cycle if and only if it belongs to a short cycle.

We need the notion of a one-point extension algebra. Let $\Lambda$ be a finite-dimensional $k$-algebra and $M$ in $\bmod \Lambda$. The one-point extension algebra $\Lambda[M]$ of $\Lambda$ by $M$ is by definition the finite dimensional $k$-algebra

$$
\Lambda[M]=\left(\begin{array}{cc}
k & 0 \\
M & \Lambda
\end{array}\right)
$$

If $\Delta=\Lambda[M]$ is the one-point extension algebra of $\Lambda$ by $M$ then the category of $\Delta$ modules is equivalent to the category of triples $\left(k^{t},{ }_{\Lambda} X, f\right)$, where $f: M \otimes k^{t} \rightarrow X$ is a map of $\Lambda$-modules.

It was shown in [HRS2] that a quasitilted algebra $\Delta$ is always of the form $\Lambda[M]$ for a quasitilted algebra $\Lambda$ and a $\Lambda$-module $M$. We will also need from [HRS2] that in this case the indecomposable direct summands of $M$ are contained in $\mathcal{L}$. Moreover the following results are established in [HRS2], Lemmas 2.1 and 2.2.

Lemma 1.3. Let $\Lambda$ be a k-algebra with $\operatorname{gl} \cdot \operatorname{dim} \Lambda \leq 2$ and let $\Delta=\Lambda[M]$ for a $\Lambda$-module $M$. Let ${ }_{\Delta} Y=\left(k^{t},{ }_{\Lambda} X, f\right)$ be in $\bmod \Delta$. Then

(i) If ker $f$ is not projective, then $\operatorname{pd}_{\Delta} Y \geq 2$.

(ii) Assume that $\operatorname{pd}_{\Lambda}$ coker $f \leq 1$. Then $\operatorname{pd}_{\Delta} Y \leq 1$ if and only if $\operatorname{ker} f$ is projective.

(iii) $\operatorname{id}_{\Delta} Y \leq 1$ if and only if $\operatorname{id}_{\Lambda} X \leq 1$ and $\operatorname{Ext}_{\Lambda}^{1}(M, X)=0$.

The following observations will be useful in the next section.

Lemma 1.4. Let $\Lambda$ be a k-algebra and let $\Delta=\Lambda[M]$ for a $\Lambda$-module $M=M_{1} \oplus M_{2}$ with $M_{1} \neq 0 \neq M_{2}$. Let $X_{1}, X_{2}$ be two indecomposable nonisomorphic $\Lambda$-modules and $f_{i}: M_{i} \rightarrow X_{i}$ be non-zero maps for $i=1,2$. Let ${ }_{\Delta} Y=\left(k,{ }_{\Lambda}\left(X_{1} \oplus X_{2}\right), f=\right.$ $\left.\left(\begin{array}{cc}f_{1} & 0 \\ 0 & f_{2}\end{array}\right)\right)$ be in $\bmod \Delta$. Then $Y$ is indecomposable.

Proof. Indeed, if $Y$ is decomposable then there exists $i$ such that $\left(0, X_{i}, 0\right)$ is a direct summand of $Y$. We may assume that $i=1$. This gives rise to the following 
commutative diagram of $\Lambda$-modules:

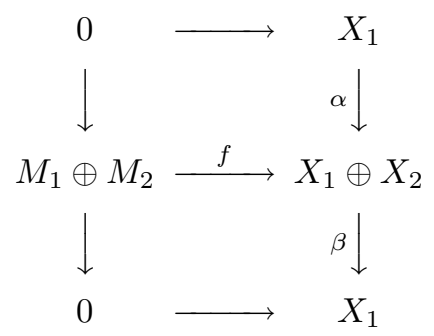

with $f \beta=0$ and $\alpha \beta=1_{X_{1}}$. Writing $\alpha=\left(\alpha_{1}, \alpha_{2}\right)$ and $\beta=\left(\begin{array}{c}\beta_{1} \\ \beta_{2}\end{array}\right)$ we obtain $f_{1} \beta_{1}=0=f_{2} \beta_{2}$ and $\alpha_{1} \beta_{1}+\alpha_{2} \beta_{2}=1_{X_{1}}$. Since $X_{1}$ is indecomposable and $X_{1} \nsucceq X_{2}$ we infer that $\alpha_{2} \beta_{2}$ is nilpotent, thus $\alpha_{1} \beta_{1}=1_{X_{1}}-\alpha_{2} \beta_{2}$ is invertible. In particular $\beta_{1}$ is invertible, and therefore $f_{1}=0$, contrary to our assumption. Thus $Y$ is an indecomposable $\Delta$-module.

Lemma 1.5. Let $\Lambda$ be a $k$-algebra and let $\Delta=\Lambda[M]$ for a $\Lambda$-module $M=M_{1} \oplus M_{2}$ with $M_{1} \neq 0 \neq M_{2}$. Let $X$ be an indecomposable $\Lambda$-module and $f_{i}: M_{i} \rightarrow X$ be maps for $i=1,2$ which are not both equal to zero. Let ${ }_{\Delta} Y=\left(k,{ }_{\Lambda} X, f=\left(\begin{array}{l}f_{1} \\ f_{2}\end{array}\right)\right)$ be in $\bmod \Delta$. Then $Y$ is indecomposable.

Proof. Indeed, if $Y$ is decomposable then we have that $(0, X, 0)$ is a direct summand of $Y$. This gives rise to following commutative diagram of $\Lambda$-modules:

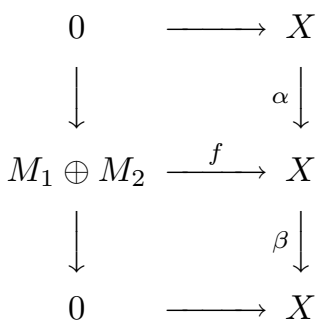

where $\alpha$ and $\beta$ are isomorphisms and $f_{1} \beta=f_{2} \beta=0$. Thus $f_{1}=f_{2}=0$ contrary to our assumption. Thus $Y$ is an indecomposable $\Delta$-module.

In the proof of the main result we will make use of the criterion in [DP]. For the convenience of the reader we will recall this result. Before doing so, we have to introduce some further notation. Recall that we may define for a finite-dimensional $k$-algebra $\Lambda$ the quiver $Q(\Lambda)$. The vertices of $Q(\Lambda)$ are the isomorphism classes $[S]$ of simple $\Lambda$-modules $S$, and the number of arrows from $\left[S^{\prime}\right]$ to $[S]$ is the dimension of $\operatorname{Ext}_{\Lambda}^{1}\left(S, S^{\prime}\right)$. We also consider a partial order on the vertices of $Q(\Lambda)$ by defining $a \preccurlyeq b$ if there is a path in $Q(\Lambda)$ from $a$ to $b$. Note that this implies that there is a path in ind $\Lambda$ from $P(a)$ to $P(b)$, where for a vertex $c \in Q(\Lambda)$ we have denoted by $P(c)$ the projective cover of the simple $\Lambda$-module $S(c)$ corresponding to the vertex $c$. Given any $\Lambda$-module $N$, we define the support algebra of $N$ as the factor algebra of $\Lambda$ modulo the ideal generated by all idempotents which annihilate $N$. Given a vertex $a \in Q(\Lambda)$, we define $\Lambda^{a}$ as the support algebra of $\underset{a \npreceq b}{\oplus} S(b)$. If $Q(\Lambda)$ has no oriented cycle we infer that the radical $\operatorname{rad} P(a)$ of $P(a)$ is an $\Lambda^{a}$-module. Given $a \in Q(\Lambda)$, we denote by $\operatorname{rad} P(a)=\oplus_{i=1}^{n_{a}} R_{i}(a)$ the decomposition of $\operatorname{rad} P(a)$ into 
indecomposable direct summands. Using this notation we have the following result from [DP].

Theorem 1.6. Let $\Lambda$ be a finite-dimensional algebra whose quiver $Q(\Lambda)$ has no oriented cycle. Then the Auslander-Reiten quiver $\Gamma_{\Lambda}$ has a preprojective component if and only if for every vertex $a \in Q(\Lambda)$ one of the following conditions is satisfied:

(i) There is a preprojective component $\mathcal{P}$ of $\Gamma_{\Lambda^{a}}$ such that $R_{i}(a) \notin \mathcal{P}$ for every $1 \leq i \leq n_{a}$.

(ii) For each $1 \leq i \leq n_{a}$ the set of predecessors $\left\{Y \in \Gamma_{\Lambda^{a}} \mid Y \preccurlyeq R_{i}(a)\right\}$ of $R_{i}(a)$ in ind $\Lambda^{a}$ is finite and consists of directing modules. If $a$ is a sink, then $\operatorname{rad} P(a)$ is a directing module in $\bmod \Lambda^{a}$.

\section{THE MAIN RESULT}

We keep the notation from the previous sections.

Lemma 2.1. Let $\Lambda$ be a quasitilted algebra and $M=M_{1} \oplus M_{2}$ a $\Lambda$-module such that $\Delta=\Lambda[M]$ is a quasitilted algebra. Then each indecomposable direct summand of $M_{1}$ is contained in $\mathcal{R}(\Lambda)$ or $M_{2}$ is projective.

Proof. Suppose that there exists an indecomposable direct summand $M_{1}^{\prime}$ of $M_{1}$ with $M_{1}^{\prime} \notin \mathcal{R}(\Lambda)$ and that $M_{2}$ is not projective. Consider the $\Delta$-module $Y=\left(k, M_{1}^{\prime}, \pi_{1}^{\prime}\right)$ where $\pi_{1}^{\prime}$ is the projection onto $M_{1}^{\prime}$. By Lemma 1.5 we have that $Y$ is indecomposable and by 1.3 we have that $\operatorname{pd}_{\Delta} Y=2$. Thus there exists an indecomposable injective $\Delta$-module ${ }_{\Delta} I$ such that $\operatorname{Hom}_{\Delta}\left(I, \tau_{\Delta} Y\right) \neq 0$. Therefore there exists a path $(I, \tau Y, E, Y)$ in ind $\Delta$, where $E$ is an indecomposable direct summand of the middle term of the Auslander-Reiten sequence ending in $Y$. Since $M_{1}^{\prime} \notin \mathcal{R}(\Lambda)$, there is a path in ind $\Lambda$ from $M_{1}^{\prime}$ to an indecomposable $\Lambda$-module $X$ with $\operatorname{id}_{\Lambda} X=2$. In particular, $X \in \mathcal{L}(\Lambda)$. By Lemma 1.2 there is a path $M_{1}^{\prime} \stackrel{f}{\longrightarrow} F \stackrel{g}{\longrightarrow} X$ in ind $\Lambda$. If $f g \neq 0$, then, by Lemma 1.3 , the indecomposable $\Delta$-module $\left(k, X,\left(\begin{array}{c}\hat{f g} \\ 0\end{array}\right)\right)$ has both projective dimension and injective dimension equal to two, a contradiction. Thus $f g=0$. Since $\operatorname{id}_{\Lambda} X=2$, we infer that $\operatorname{Hom}_{\Lambda}\left(\tau_{\Lambda}^{-} X,{ }_{\Lambda} P\right) \neq 0$ for some indecomposable projective $\Lambda$-module $P$. The following commutative diagram of $\Lambda$-modules shows that there exists a path in ind $\Delta$ from $Y$ to $(0, X, 0)$ :

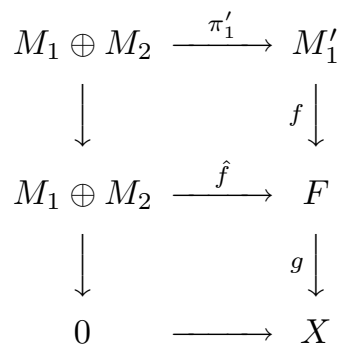

where $\hat{f}$ is the extended map as defined in the introduction. We thus obtain by combining the constructed paths a non-sectional path in ind $\Delta$ from $I$ to ${ }_{\Lambda} P$. Since $P$ is also $\Delta$-projective, we have a contradiction to 1.1 .

We also point out the following easy consequence. 
Corollary 2.2. Let $\Lambda$ be a quasitilted algebra and $M=\oplus_{i}^{t} M_{i}$ a $\Lambda$-module with $t \geq 2$ such that $\Delta=\Lambda[M]$ is a quasitilted algebra. If $M_{i} \notin \mathcal{R}(\Lambda)$ for all $1 \leq i \leq t$, then $M$ is projective.

Theorem 2.3. Let $\Delta$ be a quasitilted algebra. Then the Auslander-Reiten quiver of $\Delta$ has a preprojective component.

Proof. We may assume that $\Delta$ is not tilted. In this case $\mathcal{L} \backslash \mathcal{R}$ is a union of components of $\Gamma_{\Delta}$ by [CS]. In particular these components do not contain any indecomposable injective $\Delta$-module.

We will prove the theorem by induction on the number $n$ of simple $\Delta$-modules. For $n=1$ there is nothing to show. So assume that all quasitilted algebras with less than $n$ simple modules have a preprojective component.

Let $\Delta$ be a quasitilted algebra with $n$ simple modules. Let $Q(\Delta)$ be the quiver of $\Delta$. Let $a \in Q(\Delta)$ be a vertex. If $a$ is not a sink, then there exist a sink $\omega$ and a path from $a$ to $\omega$. Let $M=\operatorname{rad} P(\omega)$. Then there exists a quasitilted algebra $\Lambda$ such that $\Delta=\Lambda[M]$ and also $\Delta^{a}=\Lambda^{a}$. By induction, $\Lambda$ has a preprojective component, so the vertex $a$ satisfies one of the conditions in 1.6. Thus we are left with the case that $a=\omega$ is a sink. As noted before, we can write $\Delta=\Lambda[M]$ for a quasitilted algebra $\Lambda$ and a $\Lambda$-module $M=\operatorname{rad} P(\omega)$. By induction we have that the Auslander-Reiten quiver of $\Lambda$ has a preprojective component. We will show that $\omega$ satisfies one of the conditions of 1.6.

For this let $M_{1}$ be the direct sum of those indecomposable direct summands of $M$ which are contained in the preprojective components of $\Gamma_{\Lambda}$. Then $M=$ $M_{1} \oplus M_{2}$. If $\mathcal{P}$ is a preprojective component of $\Gamma_{\Lambda}$ we may assume that $\mathcal{P}$ contains an indecomposable summand of $M_{1}$. Otherwise, $\mathcal{P}$ will also be a preprojective component for the Auslander-Reiten quiver of $\Delta$; compare [DP]. In particular we will assume from now on that $M_{1} \neq 0$ and that $\omega$ does not satisfy condition (i) of 1.6 .

We will show first that $M_{2}$ is projective.

If $M_{2}$ is not projective, there exists an indecomposable non-projective direct summand $M_{2}^{\prime}$ of $M_{2}$. Let $\Lambda_{1}$ be the connected component of $\Lambda$ supporting $M_{2}^{\prime}$. We first consider the case that all indecomposable projective $\Lambda_{1}$-modules are contained in preprojective components of $\Gamma_{\Lambda_{1}}$. Let $P$ be an indecomposable projective $\Lambda_{1}$ module with $\operatorname{Hom}_{\Lambda_{1}}\left(P, \tau_{\Lambda_{1}} M_{2}^{\prime}\right) \neq 0$. By assumption we have that $P$ is contained in a preprojective component $\mathcal{P}$ which also contains an indecomposable direct summand $M_{1}^{\prime}$ of $M_{1}$. Since $\operatorname{Hom}_{\Lambda_{1}}\left(P, \tau_{\Lambda_{1}} M_{2}^{\prime}\right) \neq 0$ and $M_{2}^{\prime} \notin \mathcal{P}$, there exists $X \in \mathcal{P}$ with $M_{1}^{\prime} \preccurlyeq X$ and $\operatorname{Hom}_{\Lambda_{1}}\left(X, \tau_{\Lambda_{1}} M_{2}^{\prime}\right) \neq 0$. In particular we obtain a path from $M_{1}^{\prime}$ to $\tau_{\Lambda_{1}} M_{2}^{\prime}$. By Lemma 1.2 there exist an indecomposable $\Lambda_{1}$-module $Y$ and a path $M_{1}^{\prime} \stackrel{f}{\longrightarrow} Y \stackrel{g}{\longrightarrow} \tau M_{2}^{\prime}$ in ind $\Lambda_{1}$. We consider the $\Delta$-module $Z=\left(k, Y, \alpha=\left(\begin{array}{l}\hat{f} \\ 0\end{array}\right)\right)$. Then $Z$ is indecomposable and by Lemma 1.3 we infer that $\operatorname{pd}_{\Delta} Z=2=\operatorname{id}_{\Delta} Z$. For this note that $M_{2}^{\prime}$ is a direct summand of $\operatorname{ker} \alpha$ and $\operatorname{Ext}_{\Lambda_{1}}^{1}(M, Y) \neq 0$, for $\operatorname{Hom}_{\Lambda_{1}}(Y, \tau M) \neq 0$. This contradicts the fact that $\Delta$ is quasitilted. Therefore there exists an indecomposable projective $\Lambda_{1}$-module which is not contained in a preprojective component of $\Gamma_{\Lambda_{1}}$. Since $\Lambda_{1}$ is connected, there exist indecomposable projective $\Lambda_{1}$-modules $P, P^{\prime}$ such that $\operatorname{Hom}_{\Lambda_{1}}\left(P, P^{\prime}\right) \neq 0$ and $P$ is contained in a preprojective component $\mathcal{P}$ of $\Gamma_{\Lambda_{1}}$ and $P^{\prime}$ is not contained in a preprojective component of $\Gamma_{\Lambda_{1}}$. Again there is an indecomposable direct summand $M_{1}^{\prime}$ of $M_{1}$ contained in $\mathcal{P}$. Since $M_{2}$ is not projective, we have by Lemma 2.1 that $M_{1}^{\prime} \in$ 
$\mathcal{R}(\Lambda)$. Let $0 \neq f \in \operatorname{Hom}_{\Lambda_{1}}\left(P, P^{\prime}\right)$. Then by the choice of $P, P^{\prime}$ we have that $f \in \operatorname{rad}^{\infty}\left(P, P^{\prime}\right)$. Thus for each $r \geq 1$, there exist a chain of irreducible maps

$$
P=X_{0} \stackrel{f_{1}}{\longrightarrow} X_{1} \stackrel{f_{2}}{\longrightarrow} \cdots \stackrel{f_{r}}{\longrightarrow} X_{r}
$$

and a map $g_{r}: X_{r} \rightarrow P^{\prime}$ such that $f_{1} \ldots f_{r} g_{r} \neq 0$. Choose $r$ in such a way that $\tau X_{r}$ is a successor of $M_{1}^{\prime}$. Note that $\operatorname{id}_{\Lambda} \tau X_{r}=2$. Since $\mathcal{R}(\Lambda)$ is closed under successors and $M_{1}^{\prime} \in \mathcal{R}(\Lambda)$ we infer that $\tau X_{r} \in \mathcal{R}(\Lambda)$, a contradiction. Thus $M_{2}$ is projective.

Assume that $M_{2} \neq 0$. We will show next that in this case there exists an indecomposable $\Lambda$-module $X$ with $\operatorname{id}_{\Lambda} X=2$ and $\operatorname{Hom}_{\Lambda}\left(M_{1}, X\right) \neq 0$.

By the previous part of the proof we know that $M_{2}$ is projective. Let $M_{2}^{\prime}$ be an indecomposable direct summand of $M_{2}$ and as before let $\Lambda_{1}$ be the connected component of $\Lambda$ supporting $M_{2}^{\prime}$. Let $M_{1}^{\prime}$ be an indecomposable direct summand of $M_{1}$. Then $M_{1}^{\prime}$ lies in a preprojective component $\mathcal{P}$ of $\Gamma_{\Lambda_{1}}$. We consider $\mathcal{S}\left(M_{1}^{\prime} \rightarrow\right)$, the subset of $\mathcal{P}$ consisting of those indecomposable modules $X$ for which there is a sectional path from $M_{1}^{\prime}$ to $X$.

We distinguish the following two cases.

First assume that there is no proper successor of $\mathcal{S}\left(M_{1}^{\prime} \rightarrow\right)$ which is projective. Arguing as above we find $X \in \mathcal{S}\left(M_{1}^{\prime} \rightarrow\right)$ with $\operatorname{id}_{\Lambda} X=2$. Indeed, since $\Lambda_{1}$ is connected there exist indecomposable projective $\Lambda_{1}$-modules $P, P^{\prime}$ such that $\operatorname{Hom}_{\Lambda_{1}}\left(P, P^{\prime}\right) \neq 0$ and $P$ is contained in $\mathcal{P}$ and $P^{\prime}$ is not contained in a preprojective component of $\Gamma_{\Lambda_{1}}$. By the choice of $P, P^{\prime}$ we have that $f \in \operatorname{rad}^{\infty}\left(P, P^{\prime}\right)$. Thus for each $r \geq 1$, there exists a chain of irreducible maps

$$
P=X_{0} \stackrel{f_{1}}{\longrightarrow} X_{1} \stackrel{f_{2}}{\longrightarrow} \cdots \stackrel{f_{r}}{\longrightarrow} X_{r}
$$

and a map $g_{r}: X_{r} \rightarrow P^{\prime}$ such that $f_{1} \ldots f_{r} g_{r} \neq 0$. Choose $r$ in such a way that $\tau X_{r}$ is contained in $\mathcal{S}\left(M_{1}^{\prime} \rightarrow\right)$. Note that $\operatorname{id}_{\Lambda} \tau X_{r}=2$.

Next assume that there is a proper successor $\mathcal{S}\left(M_{1}^{\prime} \rightarrow\right)$ which is projective. Consider $\Sigma=\mathcal{S}(\rightarrow P)$, the subset of $\mathcal{P}$ consisting of those indecomposable modules $X$ for which there is a sectional path from $X$ to $P$. Note that the indecomposable modules in $\tau \Sigma$ all have injective dimension two and that there is a path from $M_{1}^{\prime}$ to an indecomposable module in $\tau \Sigma$. Also note that $\tau \Sigma$ is a separating subcategory in the sense that each map from a predecessor of $\tau \Sigma$ in $\mathcal{P}$ to a module which is not a predecessor of $\tau \Sigma$ factors through $\tau \Sigma$. We consider a nonzero map $M_{1}^{\prime}$ to an indecomposable injective module. Since any path from an indecomposable injective to an indecomposable projective is sectional for a quasitilted algebra, we infer that $I$ is not a predecessor of $\tau \Sigma$. Hence there is a nonzero map from $M_{1}^{\prime}$ to an indecomposable module in $\tau \Sigma$. This proves our claim.

Next we will show that $\operatorname{Hom}_{\Lambda}\left(M_{2}, Y\right)=0$ for all $Y \in$ ind $\Lambda$ with $\operatorname{pd}_{\Lambda} Y=2$.

Suppose to the contrary that there exists an indecomposable $\Lambda$-module $Y$ with $\operatorname{pd}_{\Lambda} Y=2$ and $\operatorname{Hom}_{\Lambda}\left(M_{2}, Y\right) \neq 0$. By the previous claim there also exists an indecomposable $\Lambda$-module $X$ with $\operatorname{id}_{\Lambda} X=2$ and $\operatorname{Hom}_{\Lambda}\left(M_{1}, X\right) \neq 0$. Choose nonzero maps $f: M_{1} \rightarrow X$ and $g: M_{2} \rightarrow Y$. Consider the $\Delta$-module

$$
Z=\left(k, X \oplus Y,\left(\begin{array}{ll}
f & 0 \\
0 & g
\end{array}\right)\right) .
$$

By Lemma 1.4 we have that $Z$ is indecomposable and by Lemma 1.3 we have that $\operatorname{pd}_{\Delta} Z=2=\operatorname{id}_{\Delta} Z$, a contradiction.

It follows from this that each submodule of $M_{2}$ is projective, since otherwise the corresponding factor module would have projective dimension two. Moreover 
it follows that $M_{2}$ is directing and each indecomposable summand of $M_{2}$ has only finitely many predecessors. In fact let $X$ be an indecomposable $\Lambda$-module with $0 \neq$ $f \in \operatorname{Hom}\left(X, M_{2}^{\prime}\right)$ for an indecomposable direct summand $M_{2}^{\prime}$ of $M_{2}$. Then let $f=$ $\pi \mu$ be the canonical factorization through $B=\operatorname{im} f$. So $B$ is projective, and hence $X$ is projective and isomorphic to $B$. As a consequence of this we infer that there is no path from an indecomposable direct summand of $M_{1}$ to an indecomposable direct summand of $M_{2}$.

As a final step we will show that $M$ is directing as a $\Lambda$-module. By the previous remark it is enough to show that $M_{1}$ is directing. Suppose that there exist indecomposable direct summands $M_{1}^{\prime}$ and $M_{1}^{\prime \prime}$ of $M_{1}$ and a non-sectional path from $M_{1}^{\prime}$ to $M_{1}^{\prime \prime}$.

If $M_{1}^{\prime \prime}$ is not projective there exists a path from $M_{1}^{\prime}$ to $\tau M_{1}^{\prime \prime}$. By Lemma 1.2 there exists a path $M_{1}^{\prime} \stackrel{f}{\longrightarrow} Y \stackrel{g}{\longrightarrow} \tau M_{1}^{\prime \prime}$. Consider the indecomposable $\Delta$-module $Z=\left(k, Y,\left(\begin{array}{l}\hat{f} \\ 0\end{array}\right)\right)$, which again by Lemma 1.3 has both projective and injective dimension two. So we have that $M_{1}^{\prime \prime}$ is projective. Again by Lemma 1.2 we obtain a path $M_{1}^{\prime} \stackrel{f}{\longrightarrow} Y \stackrel{g}{\longrightarrow} M_{1}^{\prime \prime}$. We will show that there exists an indecomposable nonprojective $\Lambda$-module $W$ such that $\operatorname{Hom}_{\Lambda}\left(M_{1}^{\prime}, \tau W\right) \neq 0$ and $\operatorname{Hom}_{\Lambda}\left(W, M_{1}^{\prime \prime}\right) \neq 0$. If $f g=0$ this follows from [HR]. So suppose that $f g \neq 0$ and let $\Sigma=\mathcal{S}\left(\rightarrow M_{1}^{\prime \prime}\right)$. By assumption we have that $M_{1}^{\prime}$ is a predecessor of $\tau \Sigma$. Therefore the nonzero map $f g$ factors through a module in $\tau \Sigma$. In particular there exists an indecomposable nonprojective $\Lambda$-module $W$ such that $\operatorname{Hom}_{\Lambda}\left(M_{1}^{\prime}, \tau W\right) \neq 0$ and $\operatorname{Hom}_{\Lambda}\left(W, M_{1}^{\prime \prime}\right) \neq 0$. Since $M_{1}^{\prime \prime}$ is projective we infer that $\operatorname{id}_{\Lambda} \tau W=2$. Let $0 \neq \alpha \in \operatorname{Hom}_{\Lambda}\left(M_{1}^{\prime}, \tau W\right)$ and $0 \neq \beta \in \operatorname{Hom}_{\Lambda}\left(W, M_{1}^{\prime \prime}\right)$. Since $W$ is non-projective we have that $\beta$ is not surjective. Also, $\operatorname{pd}_{\Lambda} \operatorname{coker} \beta=2$. Thus there exist an indecomposable $\Lambda$-module $Y$ with $\operatorname{pd}_{\Lambda} Y=2$ and a nonzero map $\gamma: M_{1}^{\prime \prime} \rightarrow Y$. Consider the indecomposable $\Delta$-module $Z=\left(k, \tau W \oplus Y,\left(\begin{array}{cc}\hat{\alpha} & 0 \\ 0 & \hat{\gamma}\end{array}\right)\right)$, which again by Lemma 1.3 has both projective and injective dimension two, a contradiction. Thus $M_{1}$, and therefore $M$, is directing.

This shows that the extension vertex $\omega \in Q(\Delta)$ satisfies the condition (ii) of Theorem 1.6. In fact, we have just seen that $M=\operatorname{rad} P(\omega)$ is directing. Also any indecomposable summand $M_{2}$ has only finitely predecessors, all of which are directing. The indecomposable direct summands of $M_{1}$ are all contained in preprojective components of $\Gamma_{\Lambda}$, and hence there are only finitely many predecessors and all are directing. Note that $\Lambda=\Delta^{\omega}$.

This finishes the proof of the theorem.

\section{EXAmple}

In this section we will consider the following example. Let $\Lambda$ be given by the path algebra of the following quiver modulo the ideal generated by all paths of length 2 :

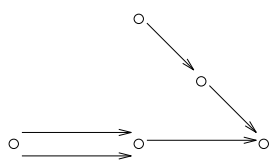


The Auslander-Reiten quiver of $\Lambda$ is given as follows. This may be used to verify the remarks below.

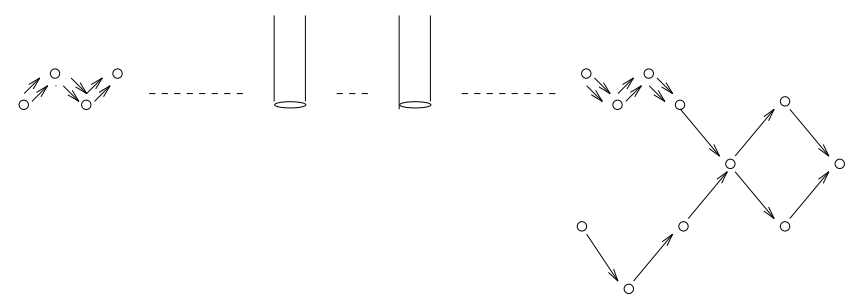

Let $S$ be the simple projective in the unique preprojective component and let $S^{\prime}$ be the simple projective in the preinjective component. Let $M$ be the sum of the two simple projective $\Lambda$-modules. We consider $\Delta=\Lambda[M]$. We claim that $\Delta$ is a quasitilted algebra. In fact, let $Z=\left(k^{t}, X, f\right)$ be an indecomposable $\Delta$-module. We may assume that $t \geq 1$; compare [HRS2]. It is easily seen that $\operatorname{Hom}_{\Lambda}(M, Y)=$ $0=\operatorname{Ext}_{\Lambda}^{1}(Y, M)$ for all indecomposable $\Lambda$-modules $Y$ with $\operatorname{pd}_{\Lambda} Y=2$. Since $Z$ is indecomposable, it follows from Lemma 1.3 that $\operatorname{pd}_{\Delta} Z \leq 1$. Thus $\Delta$ is a quasitilted algebra, and hence has a preprojective component. This of course may be verified by a direct computation. Note that $\Delta$ is a tilted algebra, for the preinjective component contains a complete slice.

\section{REFERENCES}

[ARS] Auslander, M.; Reiten, I.; Smalø, S., Representation theory of Artin algebras, Cambridge University Press 36 (1995). MR 96c: 16015

[B] Bongartz, K., A criterion for finite representation type, Math. Ann. 269 (1984), 1-12. MR 86k: 16023

[CS] Coelho, F.U.; Skowronski, A., On the Auslander-Reiten quiver of a quasitilted algebra, Fundamenta Mathematica 149 (1996), 67-82.

[DP] Dräxler, P.; de la Peña, J.A., On the existence of postprojective components in the Auslander-Reiten quiver of an algebra, preprint.

[HR] Happel, D.; Ringel, C.M., Directing projective modules, Archiv Math. 60 (1993), 247-243. MR 94b:16016

[HRS1] Happel, D.; Reiten, I.; Smalø, S., Short cycles and sincere modules, CMS Conference Proceedings 14 (1993), 233-236. CMP 94:09

[HRS2] Happel, D.; Reiten, I.; Smalø, S., Tilting in abelian categories and quasitilted algebras, Mem. Amer. Math. Soc. 120 (1996), no. 575. CMP 96:08

[R] Ringel, C.M., Tame algebras and integral quadratic forms, Springer Lecture Notes 1099 (1984). MR 87f: 16027

[S] Skowronski, A., Tame quasitilted algebras, preprint.

[St] Strauss, H, The perpendicular category of a partial tilting module, J. Algebra 144 (1991), 43-66. MR 92m:16013

Instituto de Matemática, Universidade de São Paulo, Caixa Postal 66281, São Paulo, 05389-970, BRASIL

E-mail address: fucoelho@ime.usp.br

Fakultät für Mathematik, Technische Universität Chemnitz, Postfach 964, 09107 Chemnitz, Germany

E-mail address: dieter.happel@mathematik.tu-chemnitz.de 